\title{
Affording Visual Causal Epistemologies in Epidemiology
}

\author{
Jordi Vallverdú* \\ Philosophy Department, Universitat Autonoma de Barcelona, Catalonia - Spain
}

Received: November 14, 2017; Published: December 01, 2017

*Corresponding author: Jordi Vallverdú, Room B7/104, Philosophy Department, Barcelona University, UAB, 08193 Bellaterra (BCN), Catalonia, Spain, Email: jordi.vallverdu@gmail.com

\section{Mini Review}

One of the challenges of $21^{\text {st }}$ Century sciences is how to deal with and manage huge amounts of raw data [1] Using several computational tools, scientists are able to capture, process and, finally, to understand that data. The visual aspects of this understanding process are of the utmost importance due to the specific cognitive mechanisms that make possible human thinking [2]. Epidemiology is a very complex research field devoted to the study of health and the causes of illness [3]. The difficulty of establishing sound statistical relationships between sets of events and some causal outcomes [4] has been the main source of debates within the field [5-7] Although epidemiologists and physicians have tried to avoid philosophical debates [8] about causality, it has been impossible to not be aware of the intrinsic and insurmountable problem of working with so complex amounts of data. From the simple one-hit paradigm of early epidemiology $[9,10]$ to current multi-causal webs of determinants [11], new challenges have emerged. A possible solution for the management of such sets of data has been to invest into visual causal methods: directed acyclic graphs (henceforth, DAG). These methods have allowed a visual quantitative approach to epidemiology [12-14] that fits perfectly with the current research trends in cognitive sciences which defend the power of extended and enhanced ways of using informational tools, which afford new and sound ways of processing information.

In this brief mini report I suggest several interesting corollaries of the implementation of such techniques:

a. The necessity of the enhancement of visual argumentative methods in scientific practices. A few examples, such as Venn diagrams or Feynman's diagrams $[15,16]$ have still not reached a full implementation of Western scientific cultures dominated by classic written methods.

b. The power that these visual methods give to not only human analysis but to the more fruitful interactions between human thinking and machine thinking in data-mining processes [17].

c. The epistemic reliability and soundness of visual reasoning, beyond the classic critics to these methods. d. The necessity of more analysis on the culturally embedded values into visual thinking (related on traditions on spatial uses during writing processes, to prevent some possible mistakes as well as to define better this new thinking space [18-20].

Taking into account these ideas, we'll be able to define a new reasoning scenario for epidemiological researchers that could even be exported to other domains, not only from classic sciences but also to social sciences or even humanities. This new visual quantitative methodologies may make possible a new set of scenarios for the advancement of knowledge thanks to the implementations of tools which impulse the cognitive creativity, closer to naturalized and easily affordable ways to deal with information .

\section{References}

1. Casacuberta D, Vallverdú J (2014) E-science and the data deluge. Philos Psychol 27(1): 126-140.

2. Vallverdú J (2010) Seeing for knowing: The Thomas Effect and computational science, In: Thinking Machines and the Philosophy of Computer Science: Concepts and Principles. pp. 280-293.

3. Vallverdú (2016) Causalidad y epidemiología, In: Filosofía de la epidemiología social, À Puyol, A Estany (Eds.), Madrid: Plaza y Valdés \& CSIC, Spain, pp. 97-116.

4. Vallverdú J (2016) Bayesians Versus Frequentists. Berlin Heidelberg, Berlin, Germany.

5. Lipton R, Ødegaard T (2005) Causal thinking and causal language in epidemiology: it's in the details. Epidemiol Perspect Innov 2: 8.

6. La Bastide-van Gemert S, Stolk RP, Van Den Heuvel ER, Fidler V (2014) Causal inference algorithms can be useful in life course epidemiology. J Clin Epidemiol 67(2): 190-198.

7. Morabia A (2013) Hume, Mill, Hill, and the sui generis epidemiologic approach to causal inference. Am J Epidemiol 178(10): 1526-1532.

8. Broadbent A (2013) Philosophy of Epidemiology. London: Palgrave Macmillan, UK.

9. Susser M, Susser E (1996) Choosing a future for epidemiology: I. Eras and paradigms. Am J Public Health 86(5): 668-673.

10. Susser M, Susser E (1996) Choosing a future for epidemiology: II. From black box to Chinese boxes and eco-epidemiology. Am J Public Health 86(5): 674-677. 
11. Krieger N (1994) Epidemiology and the web of causation: Has anyone seen the spider? Soc Sci Med 39(7): 887-903.

12. Daniel RM, De Stavola BL, Vansteelandt S (2016) Commentary: The formal approach to quantitative causal inference in epidemiology: Misguided or misrepresented? International Journal of Epidemiology 45(6): 1817-1829.

13. Vandenbroucke JP, Broadbent A, Pearce N (2016) Causality and causal inference in epidemiology: the need for a pluralistic approach. Int J Epidemiol 45(6): 1-11.

14. Krieger N, Smith GD (2016) The tale wagged by the DAG: Broadening the scope of causal inference and explanation for epidemiology. Int J Epidemiol 45(6): 1787-1808.
15. Hamburger $P$ (2008) Cogwheels of the mind. The story of venn diagrams. Math Intell 27(4): 36-38.

16. Ellis JP (2016) TikZ-Feynman: Feynman diagrams with TikZ. Comput Phys 210: 103-123.

17. Chen Y, Kao H, Ko M (2004) Mining DAG Patterns from DAG Databases. Adv Web-Age Inf Manag 579-588.

18. Bednarz RS, Lee J (2011) The components of spatial thinking: Empirical evidence. In: Procedia-Social and Behavioral Sciences 21: 103-107.

19. Menary R (2007) Writing as thinking. Lang Sci 29(5): 621-632.

20. Csikszentmihalyi M (2014) The systems model of creativity: The collected works of Mihaly Csikszentmihalyi.

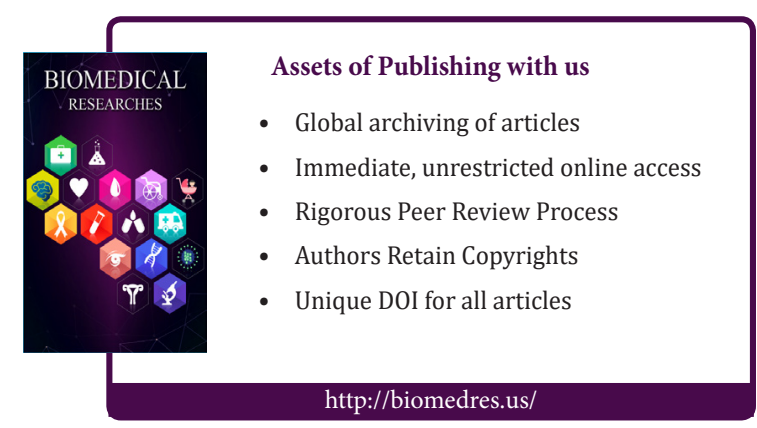

